\title{
Renaissance of Bohr's Model Via Derived Alternative Equation
}

\author{
Ikechukwu Iloh Udema ${ }^{1,2}$ \\ ${ }^{1}$ Ude International Concepts Ltd., Agbor, Nigeria \\ ${ }^{2}$ Department of Chemistry and Biochemistry, Owa Alizomor Sec. Sch., Owa Alizomor, Nigeria
}

Email address:

udema_ikechukwu99@yahoo.com

\section{To cite this article:}

Ikechukwu Iloh Udema. Renaissance of Bohr's Model Via Derived Alternative Equation American Journal of Modern Physics. Vol. 6, No. 2, 2017, pp. 23-31. doi: 10.11648/j.ajmp.20170602.11

Received: March 2, 2017; Accepted: March 11, 2017; Published: March 28, 2017

\begin{abstract}
The aims of this research were: 1) To derive alternative equation into which can be substituted known experimental data and known physical constants for the calculation of Bohr's radii of atoms for some elements, 2) respond to some of the objections raised against (or the short coming of Bohr's theory,) and 3) make a case for the justification of Bohr's theory. Apart from other elements, Bohr's radius $\left(a_{0}\right)$ for hydrogen was $0.5291 \AA$; the radii for $\mathrm{Na}\left([\mathrm{Ne}] 3 \mathrm{~s}^{1}\right)$ and $\mathrm{Na}{ }^{+}\left([\mathrm{Ne}] 3 \mathrm{~s}^{0}\right)$ were $2.5844 \AA$ and $0.5675 \AA$ respectively which correspond to effective nuclear charges $\left(Z_{\text {eff }}\right)$ equal to 1.8424 and 3.7291 respectively at the $1^{\text {st }}$ and $2^{\text {nd }}$ principal quantum numbers $(n)$ respectively. The results were obtained based on two definitions: $a_{0} \alpha n^{2} / Z_{\text {eff }}$ (from initial Bohr's equation) and $a_{0} \alpha n /\left(\xi_{\mathrm{n}}\right)^{1 / 2}$ (from the derived equation, where $\xi_{\mathrm{n}}$ is the average ionization energy). In conclusion, an alternative equation to Bohr's equation was successfully derived. No single model should always be a solution to all scientific questions. Both original Bohr's equation and derived equation can give, after calculation, similar value of any atomic radius. Therefore, Bohr's theory stands scientifically justified.
\end{abstract}

Keywords: Bohr's Equation, Heisenberg Principle, Schrödinger-Dirac Formalism, Derived Equation,

Effective Nuclear Charge, Radius

\section{Introduction}

The year, 2016, is about a 100 years after Bohr published his famous model for hydrogen atom in particular. Literature $[1,2]$ is loaded with very reprehensible comments on Bohr's model. Some comments are however, characteristically scientific in content with the intention of further comprehension of the basis or justification for the claim made by Bohr, with regard to his theory of atomic structure using hydrogen atom as a case study. The problem has always been that Bohr's contemporary and subsequent "nononsense" scientist had greater expectation from the work of Bohr as if a single model could be a solution to every question or problem connected with any phenomenon or uncomprehended observation. Doing so is like the action of highly advanced medical doctor who prescribes the same medicine for any disease: However, this is not to say that some antibiotics may not possess broad spectrum of therapeutic action. Modern approach in the description of hydrogen atom and its electron in particular is mainly based on Schrödinger-Dirac formalism. It is too obvious that classical mechanics upon which Bohr's model is based is very different from quantum mechanics, which is predominantly mathematical in nature and used to predict the position and momentum of an electron. The focus of this paper ab initio, is on Bohr's model.

Before, consideration for the criticism of Bohr's theory, the main points-which I wish to call "tenets" - of Bohr's theory or rather his postulations as may be found in literature [3] are summarized as follows: Electrons revolve around the nucleus in stable orbits without emission of radiant energy. Each orbit has a definite energy and is called energy shell or energy level. An orbit or energy level is designated as $\mathrm{K}, \mathrm{L}$, $\mathrm{M}, \mathrm{N}$ shells [4]. When the electron is in the lowest energy level, it is said to be in the ground state. An electron emits or absorbs energy when it jumps from one orbit or energy level 
to another. When it jumps from higher energy level to lower energy level it emits energy while it absorbs energy when it jumps from lower energy level to higher energy level. The energy absorbed or emitted is equal to the difference between the energies of the two energy levels $\left(E_{1}, E_{2}\right)$ and is determined by Planck's equation.

$$
\Delta \mathrm{E}=\mathrm{E} 2-\mathrm{E} 1=\mathrm{h} v
$$

where $h$ and $v$ are the Planck's constant and the frequency respectively. "The dynamical equilibrium of the systems in the stationary states is governed by the ordinary laws of mechanics, while these laws do not hold for the transition from one state to another" [3]. This statement is considered an extraordinary statement that is not clearly understood. Further support for Bohr's theory is then on classical assumption of the quantization of angular momentum which ensures the stability of the electron rings. In addition, Bohr posited that the atomic electrons would not emit electromagnetic radiation according to classical theory; discrete nonclassical radiation should occur in accordance with the empirically derived Rydberg-Ritz combination principle (1888/1908) and the Planck-Einstein formulas (1901/1905) [5]. But there are arguments against whatever reasons given to justify Bohr's model of the atom. The objectives of this research were:1) To derive alternative equation into which can be substituted known experimental data and known physical constants for the calculation of Bohr's radii of atoms for some elements, 2) respond to some of the objections raised against (or the shortcoming of) Bohr's theory, and 3) make a case for the justification of Bohr's theory.

\subsection{Arguments Against Bohr's Atomic Theory}

Then, here come the shortcomings of Bohr's theory as follows [2]: It violates the Heisenberg uncertainty principle. The Bohr atomic model or theory considers electrons to have both a known distance from the nucleus - the radius of a circle (or orbit) - and orbit i.e. known position and momentum at the same time, which is impossible according to Heisenberg. The Bohr atomic model made correct predictions for smaller sized atoms like hydrogen, but poor spectral predictions are obtained when larger atoms are considered. It failed to explain the Zeeman's effect when the spectral line is split into several components in the presence of a magnetic field. It failed to explain the Stark effect when the spectral line gets split up into fine lines in the presence of an electric field. Here again are other concerns expressed by Zollman [2] in the history of science to the effect that "an accelerating charged particle will emit continuous radiation unlike in Bohr's model. If it did, the electron would spiral quickly into the nucleus. There was no reason for this. A radio station that broadcasts at 99.5 megahertz shakes electrons in its antenna 99,500,000 times per second. Scientists in 1913 expected that light was emitted in the same way, so an electron in an atom would vibrate to emit light. However, in the Bohr model nothing vibrated; the light just appeared as the electron changed orbits. Even worse, these "quantum jumps" in orbit seemed to occur without a cause. Once an electron was in orbit with energy higher than the lowest possible energy, it would at sometime move to a lower energy, but Bohr could not ascribe a cause to that event. Then, physicists asked: how does the electron "know" where it is going? When it leaves one orbit, it gives off a photon of light. The energy of that photon is determined by the energy that the electron will have when it lands in the lower orbit. It seems that the electron knows what will happen to it before the event is finished. And, finally, what happens to the electron in between the time it leaves one orbit and the time it gets to the second orbit?"

According to Kragh [1], for a theory to be truly convincing it had to include a dynamical mechanism that caused the phenomenon in question. Bohr's theory provided neither a mechanism, nor a physical picture of the radiation process; it merely postulated discontinuous and apparently uncaused quantum jumps while Runge in Germany opined that there was no physical explanation but was at best a computational recipe. The outer shell electron experience the shielding effect of inner electrons to different degrees according to the type of orbital electrons in the following order: $s>p>d>f$. Previous works involved the adoption of a simple graphical method for using spectral data to determine the effective nuclear charges, $Z_{\text {eff }}[6]$. Previous result showed that, the interelectronic interactions must be considered in the energy calculations because of the penetration of this single electron into the inner closed shells [6]. As a consequence of the radial probability-density distribution (a concept that may be used qualitatively since its quantitative interpretation is not too clear), the effective nuclear charge, $Z_{\text {eff }}$, depends on azimuthal quantum number, $l$ as well as on the principal quantum number, $n$. There is the issue of the shielding effect of inner electrons with a shielding constant which represents the amount of nuclear charge screened by the inner electrons on the outer $n l$ electrons given as $\sigma_{\mathrm{nl}}=Z-Z_{\mathrm{eff}}$, where $Z$ is the total nuclear charge. A quantity called the quantum defect, $m_{d}$, which accounts for the distinct capability of the valence electrons to penetrate into the atom's inner closed shells, is usually introduced. Consequently, "the effective nuclear charge is a measure of the average nuclear charge felt by the outermost electron in the various orbitals, considering the interelectronic repulsions and its penetration capability" [6].

The effective nuclear charge hereby symbolized as $Z_{\text {eff }}$ is a quantity or factor which is very important in Bohr's model with respect to multi-electron atoms. The amount of energy needed to remove an electron from its orbit based on Bohr's model is called ionization energy; it may be $1^{\text {st }}, 2^{\text {nd }}, 3^{\text {rd }}$ etc ionization energy. The subsequent ionization energy does not result from the initial position of the subsequent electron that is removed because the $1: 1$ ratio of atomic number to the number of electrons has increased - greater number of protons per electron after the removal of the $1^{\text {st }}$ electron, for instance. Thus, effect of $Z_{\text {eff }}$ on ionization energy, which is also defined as the amount of energy equal to kinetic energy needed to remove an electron from nuclear influence to an 
infinite shell $(n=\infty)$ needs to be taken into account. Therefore, so long as ionization energy is a function of the distance between any electron and the nucleus, and the nuclear charge or effective nuclear charge if there is screening effect due to the presence of inner electron(s), an alternative equation for the determination of the radius can be derived subsequently.

\subsection{Derivation of Alternative Equation to Bohr's Equation}

First, equation of Bohr's radius is stated as follows:

$$
a_{0}=\frac{n^{2} h^{2} \varepsilon 0}{\pi e^{2} m Z_{\mathrm{eff}}}
$$

where $a_{0}, n$, and $\varepsilon_{0}$ are the Bohr's radius, the energy level/principal quantum number, and the permittivity of free space respectively; $e$ and $m$ are the charge and the mass of an electron respectively.

Meanwhile, the expression (as can be found in some text books) for total energy of an electron in an orbit is [7]:

$$
E_{n}=\text { P.E }+ \text { K.E }
$$

where $E_{\mathrm{n}}$ is the total energy of the electron, and P.E and K.E (redesignated as $\xi_{\mathrm{n}}$ and being $=$ kinetic energy per mole $/ N_{\mathrm{A}}$ where $N_{\mathrm{A}}$, is the Avogadro's number) are the potential energy and the kinetic energy respectively. Meanwhile the following is known in literature $[7,8]$.

$$
\xi_{n}=\frac{Z_{\text {eff }} e^{2}}{8 \pi \varepsilon_{0} a_{0}}
$$

Equation (4) represents the electrostatic energy of attraction of the nucleus for the electron and it is equivalent to ionization energy. Substitution of Eq. (2) into Eq. (4) gives:

$$
\xi_{n}=\frac{Z_{\mathrm{eff}^{2} e^{4} m}}{8 \varepsilon 0^{2} n^{2} h^{2}}
$$

It is interesting to note that $E_{\mathrm{n}}=-\xi_{\mathrm{n}}$ [7]. Just for the sake of recognition otherwise it does not help the derivation. $Z_{\text {eff }}$ in Eq. (5) can be made subject of the formula to give:

$$
Z_{\text {eff }}=\frac{\left(8 \xi_{\mathrm{n}}\right)^{1 / 2}}{m^{1 / 2}} \frac{n h \varepsilon_{0}}{e^{2}}
$$

Resubstitution of Eq. (6) into Bohr's equation, Eq. (2), gives after rearrangement:

$$
a_{0}=\frac{n h}{\pi\left(8 m \xi_{n}\right)^{1 / 2}}
$$

The concern of this paper remains mainly issues concerning Eq. (7), as a more general equation, whose dependent variable has definition different from the well known Bohr's equation.

\section{Method}

The method which is purely theoretical, implied that the data which include ionization energies [9, 10] and known constant quantities were substituted into appropriate equations in order to calculate desired parameters, $Z_{\text {eff }}$ and $a_{0}$. Equations (6) and (7) were used to calculate $Z_{\text {eff }}$ and $a_{0}$ respectively. Equation (6) enables the use of Eq. (2) for the calculation of $a_{0}$ for multielectron atoms. With Eq. (6), the values of $Z_{\text {eff }}$ can be calculated, and substituting the results into Eq. (2), gives after calculation the radii of the atoms or ions as the case may be. Equation (7) can be used directly to calculate the radii after substituting the constant parameters and the variables, the ionization energies and principal quantum number into the equation. By substituting known values of ionization energy per electron, the corresponding principal quantum number, and constant parameters into Eq. (6), gives after calculation the values of $Z_{\text {eff. The constant }}$ parameters, as can be found in any standard text book of chemistry [8, 9] are: 1) Planck's constant, the charge of an electron, the permittivity in free space, and the mass of an electron which are respectively $6.6262 \exp (-34) \mathrm{Js}, 1.6021$ $\exp (-19) \mathrm{C}, 8.85 \mathrm{~F} / \mathrm{m}$, and 9.109 (exp-31) kg. The ionization energies are as in literature $[9,10]$. Needless to emphasize that, the equations are simple algebras that can be manipulated with less difficulty using electronic calculator at any level.

\section{Results and Discussion}

From the data in Table 1 it is clear that both original Bohr's equation (Eq. (2)) and modified equation (Eq. (7)) give the same answer only for hydrogen atom. Thus Eq. (2) cannot be used directly for multielectron atoms unlike Eq. (7). The value of $Z_{\text {eff }}$ should be known before Eq. (2) can be used. As shown in Eq. (2), $a_{0}$ is directly proportional to the square of principal quantum number and inversely proportional to the effective nuclear charge. It would be inappropriate to define Eq. (7) in the same way as Eq. (2). It is clear that $a_{0}$ is inversely proportional to the square root of the average ionization energy and directly proportional to the principal quantum number. The reason is that ionization energy which appears in Eq. (7) does not appear in Eq. (2), just as the effective nuclear charge which appears in Eq. (2) does not appear in Eq. (7); yet effective nuclear charge and ionization energy are related, being mutually dependent. Meanwhile, $Z_{\text {eff }}$ value obtained from atomic spectrum for sodium at its $3^{\text {rd }}$ energy level is very similar to the calculated value using the $1^{\text {st }}$ ionization energy per mole. Besides, the older equation and its modified form give the same value of Bohr's radius for hydrogen (Table 1).

One of several characteristics of Bohr's model is its mathematical simplicity. This has been acknowledged elsewhere [3]. This may be understood in terms of the fact that what may be called formal language of science, mathematics must not always present complexities as yardstick for presumed superior contribution to solution(s) to scientific problem. No wonder therefore, Schrödinger, had the uncommon humility, to feel repelled by Heisenberg's matrix he called "transcendental algebra" that lacks visual 
connection [2]. Therefore, opinion that, very strong mathematics, calculus, often characteristics of recent approaches in the solution to atomic structure problems, is not comprehensible to many in the field, cannot be an exception. Bohr's equation is not a complex algebra. It shows a relationship between the radius of an atom and effective nuclear charge (the radius is inversely proportional to the effective nuclear charge and directly proportional to the square of principal quantum number as implied in the old model); the derived model shows that the radius of the atom is inversely proportional to the square root of average ionization energy and directly proportional to the principal quantum number.

Going by the criticism against Bohr's theory or equation, one may feel that the critics feel that Bohr's approach should have been able to provide answers to questions regarding various unexplained physical phenomenon such as emission of light, Zeeman effects etc during Bohr's days. But be it known or unknown to Bohr and the critics, Bohr's equation has potential to answer few questions; in particular, what the radius of an atom may be given experimentally or theoretically determined ionization energy. What may have been referred to as shortcomings or unanswered questions arising from certain observation about hydrogen atom or more complex atom should rather become research problems deserving investigation.

According to Heisenberg as cited in the work of Busch et al [11], "it is impossible to prepare states in which position and momentum are simultaneously well localized; it is impossible to measure position without disturbing momentum, and vice versa". This is most probable because, an electron is too small to see, and in order to "see" it has to be perturbed by hitting it with another particle [8]. Therefore, Heisenberg's position is as it affects perturbed electron which as such becomes too evasive such that the exert location and speed cannot be determined at the same time. This is clearly unlike Bohr's case in which known or unknown to him, it is the atom in its ground state that is referred to. Therefore, Heisenberg's law is not violated and may not be precluded if an excited atom only is under consideration. By the way, it must be mentioned that hydrogen atom does not exist in nature unlike helium; special instrumentation is needed to keep hydrogen atoms which are chemically unstable: This must be seen to be different from physical stability of the atom. He is physically and chemically stable because of inertness. Hydrogen atoms may be produced in the presence of electric spark and the electric aftershock can raise the electron to higher energy level. This may be the unnatural environment of the atom in which it is studied. When the effect of the electric spark gradually subsides, the momentum of the electron may decrease and it becomes strongly affected by the nuclear charge. The removal of electrons to infinite shell requires very high voltage. If electric spark intensity decreases, it may lead to lose of momentum of escaping electron; this may either bring the electron to initial energy level or to the nucleus in an electron capture process.

Bohr's model for an atom like hydrogen merely addresses some aspects of many physical phenomena, the energies of electrons in any atom accounted for on the basis of the distance between the nucleus and the electron domicile in different energy levels; hence an electron has potential energy due to its position relative to the nucleus and kinetic energy due to its attraction by the nucleus. Zeeman and Stark effects cannot occur in the absence of magnetic and electric fields respectively. If on the contrary, then an interpretation with different model may be needed.

A betatron is a type of particle accelerator that uses the electric field induced by a varying magnetic field to accelerate electrons (beta rays) to high speeds in a circular orbit. In cathode ray tube experiment cathode rays can accelerate if the voltage is increased and they can be made to deflect at varying speed. Perhaps these examples are different from orbital motion of any outer electron which is under attractive influence of centrally located nucleus unlike the former devices stated. "From the electrodynamic point of view, moving electrons would radiate and lose energy" [5]. It is not certain if there had been any report which shows that the positive nucleus contributes to the loss of energy. If so, orbiting electrons at very high speed in betatron may not require centrally located body with positive charge or positive electrode potential to lose energy. Then the question is what then accounts for the failure to lose energy by the orbiting electrons in betatron? The electrons can decelerate if voltage is lowered. Then the question would have been, what triggered the motion of the electrons in the first place rather than castigate Bohr's model. Particles much larger than electron are inconstant motion. Should the orbital motion of an electron be an exemption? Mere fact that an electron bears a negative charge does not imply that it is totally amenable to kinetic theory of elementary particle which indicates that molecules must be in constant motion anywhere including vacuum. Each molecule could be more than 1840-fold heavier than an electron. Recent opinion is that the resonant nature of the circulating electron and its induced magnetic and Faraday fields prevent a radiative collapse of the electron into the nuclear proton [12]. It is important to investigate the likelihood of radiations from accelerating electrons in a betatron!

If the law of conservation of energy is still valid, then the question as to why electron should move to higher energy level may be answered. If the thermal energy or other form of energy perturbs an electron, it may ascend to higher energy level without escaping the nuclear influence in totality in accordance to the quantum of energy absorbed. The energy absorbed is converted to potential energy; antenna is not a plastic material or any other material other than a metal. A true metal is known to contain mobilized valence electrons which suggest that they are not totally under the attractive influence of the nuclei. Thus external perturbation can separate the free electrons to infinity. This is very much unlike the atom whose outer electron is still strongly under the influence of the nucleus. The electromagnetic spectrum contains visible and invisible regions. Even the visible region does not exist as spectra with the same wave length even if it is 
composed of different colours of light with the same velocity. The question that seems to be asked is: Does the electron need to end its fall at the $2^{\text {nd }}$ energy level before emission of any spectrum? Then this question is as good as does the potential energy decrease as the electron fall to lower energy level? The magnitude of the energy released as the electron falls determine the energy or wavelength of the visible spectrum and any other spectrum.

Unavoidability of Heisenberg principle is mainly applicable to perturbed atom. The effective nuclear charge which is less than the total charge in the nuclear environment of multielectron atoms mainly but not exclusively, determines the magnitude of ionization energy of multielectron atoms. This notwithstanding, Eq. (2), the original Bohr's equation and the derived new equation (Eq. (7)) in particular, based on several principles, are in total agreement with respect to the result obtainable from them for hydrogen atom in particular. One needs to add that with the advent of quantum theory according to Lang and Barry [13], the two particle problem can be solved exactly, and the kinetic energy of the electron in a hydrogen atom or hydrogen-like ion can be calculated using the Schrödinger equation. However, there has been research into other methods for the determination of ionization energies of atoms, one-, two, three-, four-, and five-electron ions [13, 14].

Ultimately the application of Bohr's model depends on the known ionization energy of the atom, be it $1^{\text {st }}, 2^{\text {nd }}$, etc ionization energy. It seems therefore, that the limitation in Bohr's approach is that it cannot be used directly to determine $1^{\text {st }}, 2^{\text {nd }}$, etc ionization energies of multielectron atoms. Although the position of electron in perturbed atom may not be known, the ionization energy of an atom defined as the amount of energy required to dislodge an electron from the outermost orbit of an atom [15] depends largely on the distance of its uncertain distance from the nucleus. While there may be other factors including electron-electron interaction which diminishes the nuclear effect [13], the focus of this research is mainly on the determination of the radius of any atom using the derived new model. Equation 7 is more straightforward in its application because only one experimental variable, the ionization energy per mole, needs to be determined if multielectron atom is the case. However, ionization energy cannot be described as independent variable because it depends on $Z_{\mathrm{eff}}$ which in turn depends on the distance of outer electron from the nucleus, the number of inner orbital electrons, and in particular, the type of orbital in which the electron is located.

The validity of Bohr's model is the issue in contention. This is vis-à-vis, what has been called the degenerative phase of Bohr's theory which was finally replaced by the quantum theory of Schrödinger and Heisenberg in 1926 [5, 16]. So far, it appears that Bohr's radius had been applicable to only hydrogen despite the fact that the ionization energy of an atom in gas phase is partly a function of the distance between outer orbital electron and the nucleus. Research in recent time focuses on atomic radii such as covalent radii [17-19] and metallic radii [18] with entirely different methods. Thus, there is no basis for a focused research like this to compare the theoretical result for Bohr's radii from this research with values of covalent and metallic radii. This research has however, led to the renaissance of Bohr's model for the determination of the radii of atoms whose ionization energies are known. In a review report, Owolabi et al [18], observed that atom of every element has electrons which revolve round the nucleus where Newtonian mechanics is violated. However, this is despite the fact that Bohr's orbiting electron is best described according to classical mechanics opposed by quantum mechanics or Heisenberg uncertainty principle. The value reported for hydrogen was $0.5292 \AA$ that seem to suggest that in the same Table [19] the values reported for other elements, $\mathrm{He}, \mathrm{Li}, \mathrm{Be}, \mathrm{B}, \mathrm{C}, \mathrm{N}, \mathrm{O}, \mathrm{F}, \mathrm{Ne}, \mathrm{Na}, \mathrm{Fe}$, etc were for the distance between the outermost ( $n$th shell) electron and the nucleus. The values reported for $\mathrm{He}, \mathrm{Li}$, and $\mathrm{Na}$ [19] were similar to calculated values of the elements in this research.

The results for $\mathrm{Be}, \mathrm{B}$, and $\mathrm{Fe}$ [19] were similar to the radii calculated for monovalent ions of the same elements while the results for $\mathrm{C}, \mathrm{N}$, and $\mathrm{O}$ [19] were much shorter in length than values for the same elements in this research. However, one cannot be too sure about the exact parameter that was determined by Ghosh and Biswas [19]. The radii for monovalent ions reported by Ghosh and Biswas [20] were shorter than the radii reported for the corresponding elements in this research. Like other authors [18, 20], Islam and Ghosh [21] computed the spectroscopic radii of atoms using different method. The values reported for selected elements by different authors were not very similar. For instance the results [21] for $\mathrm{Li}$, $\mathrm{Be}, \mathrm{B}, \mathrm{O}$, and $\mathrm{C}$ after conversion to Angstrom unit are not however, widely different from those reported by Duchwicz and Castro[17]. The radii reported for $\mathrm{Li}, \mathrm{B}, \mathrm{C}$, and $\mathrm{O}$ by Islam and Ghosh were very similar to those reported by Owolabi et al [18]. However, the values calculated using the derived equation in this research for most of the elements were different from those reported in literature [18-21]. This is in addition to the research [18], aided with support vector machine, which has shown that the radius of hydrogen atom is shorter than Bohr's radius, the atomic unit of length. The radius calculated for $\mathrm{H}$ is very similar to atomic unit of length using the derived equation in this research while the calculated radius for $\mathrm{He}$ is longer than the calculated length for He elsewhere [21].

Thus Bohr's theory is scientifically justified along the following lines: $a_{0} \alpha n^{2} / \mathrm{Z}_{\text {eff }}$ (from initial Bohr's equation) and $a_{0} \alpha n /\left(\xi_{\mathrm{n}}\right)^{1 / 2}$ (from the derived equation). Experimental and theoretical methods for the determination of ionization energies which is a function of effective nucleus-electron attraction [22] combined with newly derived equation in this research can enhance the calculation of atomic and ionic radii. An effective nucleus-electron attraction gives kinetic energy equal to ionization energy but its description as one that is proportional to the effective nuclear charge and inversely 
proportional to the effective principal quantum number [22] seems to disagree with Eq. (5). Perhaps, the author [22] may have made a mistake. However, Islam [22] determined orbital exponents $\left(\xi_{\text {orb }}\right)$ given as $Z_{\text {eff }} / n^{*}$ where $n^{*}$ is the effective principal quantum number. It implies that $Z_{\text {eff }}$ $=n^{*} \xi_{\text {orb }}$. While this research is not concerned with the determination of ionization energy which is similar to atomic hardness defined as the attraction of the nucleus upon the outer valence electron [22], Islam and Jana [23] explored orbital exponents for the determination of ionization energy utilizing modified Bohr's equation. The authors [23] indicated that the $1^{\text {st }}$ ionization energy depends on the principal quantum number and the azimuthal quantum. This shows that Bohr's model may not be invalidated and it may remain very relevant to the determination of the usual and desired parameters given experimentally or theoretically determined variables.

Table 1. The effective nuclear charge, $1^{\text {st }}$ and $2^{\text {nd }}$ radii of selected elements.

\begin{tabular}{|c|c|c|c|c|c|}
\hline Elements & Grd.stateconfig. & $1^{\text {st }} Z_{\text {eff- }}$ & $2^{\text {nd }} Z_{\text {eff }}$ & $1^{\text {st }} a_{0} / \AA$ & $2^{\text {nd }} a_{0} / \AA$ \\
\hline Hydrogen & $1 \mathrm{~s}^{1}$ & $\sim 1.000$ & - & 0.529 & - \\
\hline Helium & $1 \mathrm{~s}^{2}$ & 1.344 & $\sim 2.000$ & 0.394 & 0.265 \\
\hline Lithium & {$[\mathrm{He}] 2 \mathrm{~s}^{1}$} & 1.259 & 2.357 & 1.681 & 0.224 \\
\hline Beryllium & {$[\mathrm{He}] 2 \mathrm{~s}^{2}$} & 1.656 & 2.313 & 1.278 & 0.914 \\
\hline Boron & {$[\mathrm{He}] 2 \mathrm{~s}^{2} 2 \mathrm{p}^{1}$} & 1.561 & 2.718 & 1.355 & 0.778 \\
\hline Carbon & {$[\mathrm{He}] 2 \mathrm{~s}^{2} 2 \mathrm{p}^{2}$} & 1.819 & 2.676 & 1.163 & 0.790 \\
\hline Nitrogen & {$[\mathrm{He}] 2 \mathrm{~s}^{2} 2 \mathrm{p}^{3}$} & 2.066 & 2.950 & 1.024 & 0.717 \\
\hline Fluorine & {$[\mathrm{He}] 2 \mathrm{~s}^{2} 2 \mathrm{p}^{5}$} & 2.262 & 3.205 & 3.205 & 0.660 \\
\hline Neon & {$[\mathrm{He}] 2 \mathrm{~s}^{2} 2 \mathrm{p}^{6}$} & 2.517 & 3.469 & 0.840 & 0.660 \\
\hline Sodium & {$[\mathrm{Ne}] 3 \mathrm{~s}^{1}$} & 1.842 & 3.729 & 2.582 & 0.568 \\
\hline Magnesium & {$[\mathrm{Ne}] 3 \mathrm{~s}^{2}$} & 2.248 & 3.153 & 2.117 & 1.510 \\
\hline Aluminium & {$[\mathrm{Ne}] 3 \mathrm{~s}^{2} 3 \mathrm{p}^{1}$} & 1.989 & 3.528 & 2.393 & 1.349 \\
\hline Silicon & {$[\mathrm{Ne}] 3 \mathrm{~s}^{2} 3 \mathrm{p}^{2}$} & 2.321 & 3.287 & 2.050 & 1.448 \\
\hline Phosphorus & {$[\mathrm{Ne}] 3 \mathrm{~s}^{2} 3 \mathrm{p}^{3}$} & 3.412 & 3.614 & 5.752 & 4.190 \\
\hline Argon & {$[\mathrm{Ne}] 3 \mathrm{~s}^{2} 3 \mathrm{p}^{6}$} & 3.228 & 4.274 & 1.475 & 1.114 \\
\hline Potassium & {$[\mathrm{Ar}] 4 \mathrm{~s}^{1}$} & 2.258 & 4.573 & 3.746 & 1.041 \\
\hline Calcium & {$[\mathrm{Ar}] 4 \mathrm{~s}^{2}$} & 2.680 & 3.735 & 3.157 & 2.265 \\
\hline Scandium & {$[\mathrm{Ar}] 3 \mathrm{~d}^{1} 4 \mathrm{~s}^{2}$} & 2.777 & 3.878 & 3.046 & 2.181 \\
\hline Titanium & {$[\mathrm{Ar}] 3 \mathrm{~d}^{2} 4 \mathrm{~s}^{2}$} & 2.833 & 3.994 & 2.987 & 2.118 \\
\hline Vanadium & {$[\mathrm{Ar}] 3 \mathrm{~d}^{3} 4 \mathrm{~s}^{2}$} & 2.816 & 4.150 & 3.005 & 2.039 \\
\hline Chromium & {$[\mathrm{Ar}] 3 \mathrm{~d}^{5} 4 \mathrm{~s}^{1}$} & 2.820 & 3.301 & 3.000 & 1.442 \\
\hline Manganese & {$[\mathrm{Ar}] 3 \mathrm{~d}^{5} 4 \mathrm{~s}^{2}$} & 2.956 & 4.287 & 2.863 & 1.974 \\
\hline Iron & {$[\mathrm{Ar}] 3 \mathrm{~d}^{6} 4 \mathrm{~s}^{2}$} & 3.047 & 4.361 & 2.776 & 1.940 \\
\hline Cobalt & {$[\mathrm{Ar}] 3 \mathrm{~d}^{7} 4 \mathrm{~s}^{2}$} & 3.046 & 4.480 & 2.780 & 1.889 \\
\hline Nickel & {$[\mathrm{Ar}] 3 \mathrm{~d}^{8} 4 \mathrm{~s}^{2}$} & 2.996 & 4.621 & 2.824 & 1.831 \\
\hline Copper & {$[\mathrm{Ar}] 3 \mathrm{~d}^{10} 4 \mathrm{~s}^{1}$} & 3.013 & 3.662 & 2.808 & 1.299 \\
\hline Zinc & {$[\mathrm{Ar}] 3 \mathrm{~d}^{10} 4 \mathrm{~s}^{2}$} & 3.322 & 4.595 & 2.547 & 1.841 \\
\hline Gallium & {$[\mathrm{Ar}] 3 \mathrm{~d}^{10} 4 \mathrm{~s}^{2} 4 \mathrm{p}^{1}$} & 2.655 & 4.910 & 3.187 & 1.723 \\
\hline Germanium & {$[\mathrm{Ar}] 3 \mathrm{~d}^{10} 4 \mathrm{~s}^{2} 4 \mathrm{p}^{2}$} & 3.046 & 4.327 & 2.777 & 1.955 \\
\hline Bromine & {$[\mathrm{Ar}] 3 \mathrm{~d}^{10} 4 \mathrm{~s}^{2} 4 \mathrm{p}^{5}$} & 3.726 & 5.061 & 2.271 & 1.672 \\
\hline Krypton & {$[\mathrm{Ar}] 3 \mathrm{~d}^{10} 4 \mathrm{~s}^{2} 4 \mathrm{p}^{6}$} & 4.056 & 5.350 & 2.087 & 1.581 \\
\hline Rubidium & {$[\mathrm{Kr}] 5 \mathrm{~s}^{1}$} & 2.769 & 5.663 & 4.774 & 1.494 \\
\hline Strontium & {$[\mathrm{Kr}] 5 \mathrm{~s}^{2}$} & 3.234 & 4.500 & 4.088 & 2.938 \\
\hline Yttrium & {$[\mathrm{Kr}] 4 \mathrm{~d}^{1} 5 \mathrm{~s}^{2}$} & 3.379 & 4.739 & 3.912 & 2.790 \\
\hline Zirconium & {$[\mathrm{Kr}] 4 \mathrm{~d}^{2} 5 \mathrm{~s}^{2}$} & 3.490 & 3.933 & 3.788 & 2.689 \\
\hline Niobium & {$[\mathrm{Kr}] 4 \mathrm{~d}^{4} 5 \mathrm{~s}^{1}$} & 3.523 & 4.10 & 3.753 & 2.064 \\
\hline Molybdenum & {$[\mathrm{Kr}] 4 \mathrm{~d}^{5} 5 \mathrm{~s}^{1}$} & 3.609 & 4.359 & 3.663 & 1.941 \\
\hline Technetium & {$[\mathrm{Kr}] 4 \mathrm{~d}^{5} 5 \mathrm{~s}^{2}$} & 3.655 & 5.289 & 3.617 & 2.500 \\
\hline Ruthenium & {$[\mathrm{Kr}] 4 \mathrm{~d}^{7} 5 \mathrm{~s}^{1}$} & 3.676 & 4.442 & 3.596 & 1.905 \\
\hline Rhodium & {$[\mathrm{Kr}] 4 \mathrm{~d}^{8} 5 \mathrm{~s}^{1}$} & 3.701 & 4.603 & 3.572 & 1.838 \\
\hline Palladium & {$[\mathrm{Kr}] 4 \mathrm{~d}^{10}$} & 3.130 & 4.772 & 2.703 & 1.773 \\
\hline Silver & {$[\mathrm{Kr}] 4 \mathrm{~d}^{10} 5 \mathrm{~s}^{1}$} & 3.730 & 5.021 & 3.545 & 1.685 \\
\hline Cadmium & {$[\mathrm{Kr}] 4 \mathrm{~d}^{10} 5 \mathrm{~s}^{2}$} & 4.064 & 5.572 & 3.253 & 2.373 \\
\hline Indium & {$[\mathrm{Kr}] 4 \mathrm{~d}^{10} 5 \mathrm{~s}^{2} 5 \mathrm{p}^{1}$} & 3.260 & 5.886 & 4.056 & 2.246 \\
\hline Tin & {$[\mathrm{Kr}] 4 \mathrm{~d}^{10} 5 \mathrm{~s}^{2} 5 \mathrm{p}^{2}$} & 3.672 & 5.183 & 3.600 & 2.551 \\
\hline Antimony & {$[\mathrm{Kr}] 4 \mathrm{~d}^{10} 5 \mathrm{~s}^{2} 5 \mathrm{p}^{3}$} & 3.984 & 5.509 & 5.509 & 2.400 \\
\hline Tellurium & {$[\mathrm{Kr}] 4 \mathrm{~d}^{10} 5 \mathrm{~s}^{2} 5 \mathrm{p}^{4}$} & 4.067 & 5.836 & 3.250 & 2.265 \\
\hline Iodine & {$[\mathrm{Kr}] 4 \mathrm{~d}^{10} 5 \mathrm{~s}^{2} 5 \mathrm{p}^{5}$} & 4.381 & 5.927 & 3.018 & 2.231 \\
\hline Xenon & {$[\mathrm{Kr}] 4 \mathrm{~d}^{10} 5 \mathrm{~s}^{2} 5 \mathrm{p}^{6}$} & 4.719 & 6.240 & 2.801 & 2.118 \\
\hline
\end{tabular}




\begin{tabular}{|c|c|c|c|c|c|}
\hline Elements & Grd.stateconfig. & $1^{\text {st }} Z_{\text {eff. }}$ & $2^{\text {nd }} Z_{\text {eff. }}$ & $1^{\text {st }} a_{0} / \AA$ & $2^{\text {nd }} a_{0} / \AA$ \\
\hline Caesium & {$[\mathrm{Xe}] 6 \mathrm{~s}^{1}$} & 3.209 & 6.521 & 5.933 & 2.027 \\
\hline Barium & {$[\mathrm{Xe}] 6 \mathrm{~s}^{2}$} & 3.712 & 5.143 & 5.080 & 3.702 \\
\hline Lanthanum & {$[\mathrm{Xe}] 5 \mathrm{~d}^{1} 6 \mathrm{~s}^{2}$} & 3.840 & 5.407 & 4.958 & 3.521 \\
\hline Cerium & {$[\mathrm{Xe}] 4 \mathrm{f}^{2} 6 \mathrm{~s}^{2}$} & 3.827 & 5.364 & 4.975 & 3.549 \\
\hline Praseodymium & {$[\mathrm{Xe}] 4 \mathrm{f}^{3} 6 \mathrm{~s}^{2}$} & 3.800 & 5.287 & 5.009 & 3.601 \\
\hline Neodymium & {$[\mathrm{Xe}] 4 \mathrm{f}^{4} 6 \mathrm{~s}^{2}$} & 3.822 & 5.338 & 4.981 & 3.566 \\
\hline Promethium & {$[\mathrm{Xe}] 4 \mathrm{f}^{5} 6 \mathrm{~s}^{2}$} & 3.847 & 5.364 & 4.949 & 3.549 \\
\hline Samarium & {$[\mathrm{Xe}] 4 \mathrm{f}^{6} 6 \mathrm{~s}^{2}$} & 3.865 & 5.415 & 4.928 & 3.516 \\
\hline Europium & {$[\mathrm{Xe}] 4 \mathrm{f}^{7} 6 \mathrm{~s}^{2}$} & 3.872 & 5.453 & 4.917 & 3.491 \\
\hline Gadolinium & {$[\mathrm{Xe}] 4 \mathrm{f}^{7} 5 \mathrm{~d}^{1} 6 \mathrm{~s}^{2}$} & 4.034 & 5.662 & 4.721 & 3.362 \\
\hline Terbium & {$[\mathrm{Xe}] 4 \mathrm{f}^{9} 6 \mathrm{~s}^{2}$} & 3.938 & 5.515 & 4.835 & 3.452 \\
\hline Dysprosium & {$[\mathrm{Xe}] 4 \mathrm{f}^{10} 6 \mathrm{~s}^{2}$} & 3.963 & 5.565 & 4.804 & 3.421 \\
\hline Holmium & {$[\mathrm{Xe}] 4 \mathrm{f}^{11} 6 \mathrm{~s}^{2}$} & 3.990 & 5.589 & 4.771 & 3.406 \\
\hline Erbium & {$[\mathrm{Xe}] 4 \mathrm{f}^{12} 6 \mathrm{~s}^{2}$} & 4.019 & 5.614 & 4.737 & 3.391 \\
\hline Thulium & {$[\mathrm{Xe}] 4 \mathrm{f}^{13} 6 \mathrm{~s}^{2}$} & 4.044 & 5.638 & 4.708 & 3.376 \\
\hline Ytterbium & {$[\mathrm{Xe}] 4 \mathrm{f}^{14} 6 \mathrm{~s}^{2}$} & 4.066 & 5.674 & 4.682 & 3.355 \\
\hline Lutetium & {$[\mathrm{Xe}] 4 \mathrm{f}^{14} 5 \mathrm{~d}^{1} 6 \mathrm{~s}^{2}$} & 3.788 & 6.060 & 5.026 & 3.142 \\
\hline Hafnium & {$[\mathrm{Xe}] 4 \mathrm{f}^{14} 5 \mathrm{~d}^{2} 6 \mathrm{~s}^{2}$} & 4.248 & 6.282 & 4.169 & 3.031 \\
\hline Tantalum & {$[\mathrm{Xe}] 4 \mathrm{f}^{14} 5 \mathrm{~d}^{3} 6 \mathrm{~s}^{2}$} & 4.567 & 6.411 & 4.169 & 2.969 \\
\hline Tungsten & {$[\mathrm{Xe}] 4 \mathrm{f}^{14} 5 \mathrm{~d}^{4} 6 \mathrm{~s}^{2}$} & 4.594 & 6.825 & 4.144 & 2.789 \\
\hline Rhenium & {$[\mathrm{Xe}] 4 \mathrm{f}^{14} 5 \mathrm{~d}^{5} 6 \mathrm{~s}^{2}$} & 4.564 & 5.876 & 4.171 & 3.240 \\
\hline Osmium & {$[\mathrm{Xe}] 4 \mathrm{f}^{14} 5 \mathrm{~d}^{6} 6 \mathrm{~s}^{2}$} & 4.798 & 6.622 & 3.968 & 2.875 \\
\hline Iridium & {$[\mathrm{Xe}] 4 \mathrm{f}^{14} 5 \mathrm{~d}^{7} 6 \mathrm{~s}^{2}$} & 4.911 & 6.622 & 3.877 & 2.875 \\
\hline Platinum & {$[\mathrm{Xe}] 4 \mathrm{f}^{14} 5 \mathrm{~d}^{9} 6 \mathrm{~s}^{2}$} & 4.883 & 5.838 & 3.899 & 2.264 \\
\hline Gold & {$[\mathrm{Xe}] 4 \mathrm{f}^{14} 5 \mathrm{~d}^{10} 6 \mathrm{~s}^{2}$} & 4.938 & 6.135 & 3.855 & 2.154 \\
\hline Mercury & {$[\mathrm{Xe}] 4 \mathrm{f}^{14} 5 \mathrm{~d}^{10} 6 \mathrm{~s}^{2}$} & 5.253 & 7.043 & 3.624 & 2.703 \\
\hline Thallium & {$[\mathrm{Xe}] 4 \mathrm{f}^{14} 5 \mathrm{~d}^{10} 6 \mathrm{~s}^{2} 6 \mathrm{p}^{1}$} & 4.019 & 7.349 & 4.737 & 2.590 \\
\hline Lead & {$[\mathrm{Xe}] 4 \mathrm{f}^{14} 5 \mathrm{~d}^{10} 6 \mathrm{~s}^{2} 6 \mathrm{p}^{2}$} & 4.426 & 6.305 & 4.299 & 3.020 \\
\hline Bismuth & {$[\mathrm{Xe}] 4 \mathrm{f}^{14} 5 \mathrm{~d}^{10} 6 \mathrm{~s}^{2} 6 \mathrm{p}^{3}$} & 4.389 & 6.642 & 4.337 & 2.866 \\
\hline Polonium & {$[\mathrm{Xe}] 4 \mathrm{f}^{14} 5 \mathrm{~d}^{10} 6 \mathrm{~s}^{2} 6 \mathrm{p}^{4}$} & 4.717 & 4.035 & - & - \\
\hline Astatine & {$[\mathrm{Xe}] 4 \mathrm{f}^{14} 5 \mathrm{~d}^{10} 6 \mathrm{~s}^{2} 6 \mathrm{p}^{5}$} & 4.963 & 3.835 & - & - \\
\hline Radon & {$[\mathrm{Xe}] 4 \mathrm{f}^{14} 5 \mathrm{~d}^{10} 6 \mathrm{~s}^{2} 6 \mathrm{p}^{6}$} & 5.331 & 3.571 & - & - \\
\hline Francium & {$[\mathrm{Rn}] 7 \mathrm{~s}^{1}$} & 3.765 & 6.883 & - & - \\
\hline Radium & {$[\mathrm{Rn}] 7 \mathrm{~s}^{2}$} & 4.358 & 6.043 & 5.947 & 4.288 \\
\hline Actinium & {$[\mathrm{Rn}] 6 \mathrm{~d}^{1} 7 \mathrm{~s}^{2}$} & 4.314 & 6.606 & 6.006 & 3.893 \\
\hline Thorium & {$[\mathrm{Rn}] 6 \mathrm{~d}^{2} 7 \mathrm{~s}^{2}$} & 4.679 & 6.434 & 5.579 & 4.027 \\
\hline Protactinium & {$[R n] 5 f^{2} 6 d^{1} 7 s^{2}$} & 4.603 & 5.629 & - & - \\
\hline Uranium & {$[\mathrm{Rn}] 5 \mathrm{f}^{3} 6 \mathrm{~d}^{1} 7 \mathrm{~s}^{2}$} & 4.721 & 7.278 & 5.499 & 3.560 \\
\hline Neptunium & {$[R n] 5 f^{4} 6 d^{1} 7 s^{2}$} & 4.748 & - & 5.457 & - \\
\hline Plutonium & {$[\mathrm{Rn}] 5 \mathrm{f}^{6} 7 \mathrm{~s}^{1}$} & 4.670 & - & 5.549 & - \\
\hline Americium & {$[\mathrm{Rn}] 5 \mathrm{f}^{7} 7 \mathrm{~s}^{2}$} & 4.643 & - & 5.581 & - \\
\hline Curium & {$[R n] 5 f^{7} 6 d^{1} 7 s^{2}$} & 4.655 & - & 5.566 & - \\
\hline Berkelium & {$[R n] 5 f^{9} 7 s^{2}$} & 4.735 & - & 5.473 & - \\
\hline Californium & {$[\mathrm{Rn}] 5 \mathrm{f}^{10} 7 \mathrm{~s}^{2}$} & 4.762 & - & 5.441 & - \\
\hline Einsteinium & {$[\mathrm{Rn}] 5 \mathrm{f}^{11} 7 \mathrm{~s}^{2}$} & 4.805 & - & 5.393 & - \\
\hline Fermium & {$[R n] 5 f^{12} 7 s^{2}$} & 4.836 & - & 5.358 & - \\
\hline Mendelevium & {$[\mathrm{Rn}] 5 \mathrm{f}^{13} 7 \mathrm{~s}^{2}$} & 4.867 & - & 5.324 & - \\
\hline Nobelium & {$[\mathrm{Rn}] 5 \mathrm{f}^{14} 7 \mathrm{~s}^{2}$} & 4.893 & - & 5.295 & - \\
\hline Lawrencium & {$[\mathrm{Rn}] 5 \mathrm{f}^{14} 6 \mathrm{~d}^{1} 7 \mathrm{~s}^{2}$} & 4.187 & - & 6.189 & - \\
\hline
\end{tabular}

Grd.stateconfig. $Z_{\text {eff }}$, and $a_{0}$ are ground state configuration, effective nuclear charge and Bohr's radius. The values of ionization energy per mole for each of the elements in the Table can be found in literature cited $[9,10]$. For the purpose of confirmation, the $1^{\text {st }}$ ionization energies per mole, for hydrogen and sodium are 1312 and $495 \mathrm{~kJ} / \mathrm{mol}$ respectively; the $2^{\text {nd }}$ ionization energy for sodium is $4563 \mathrm{~kJ} / \mathrm{mol}$ [9]. Calculated parameters are approximated to 3 decimal places.

Although this paper is not concerned with historical development of Bohr's method or model, the reference to modern trend against it in literature, may justify an over view of modern theory which explains the wave nature of a perturbed electron of hydrogenic atoms in general. The origin of wave mechanics as applied to first, hydrogen atom, and subsequent hydrogenic atoms is as a result of the evasive nature of a perturbed electron. Such electron has to be treated as wave with suitable mathematical formalism, otherwise called Schrödinger-Dirac formalism that falls out of the scope of classical mechanics which explains Bohr's method.
Thus this statement as follows justifies this position: "The hydrogen atom wave functions and energies, we have seen, are determined as a combination of the various quantum" dynamical" analogues of classical motions (translation, vibration, rotation) and a central-force interaction (i.e., the Coulomb interaction between an electron and a nucleus)" [24]. However, it seems there is need for the recognition of Schrodinger's and Dirac's contribution (in addition to the work of Heisenberg) that advanced quantum mechanics to the description of hydrogenic atoms. Although the mathematical formalism in this issues may not be well 
known to some persons including the author of this research, there is discontent with Schrödinger model with evidence as follows: "It gives the principal energy levels of the hydrogen atom as eigenvalues of eigenfunction solutions of the Laguerre differential equation. But, as the principal quantum number $n>>1$, the eigenfunctions become nonsensical. Despite its wide acceptance, on deeper inspection, the Schrödinger equation solution is plagued with many failings as well as difficulties in terms of a physical interpretation that have caused it to remain controversial since its inception. Only the one electron atom (hydrogen and the hydrogenic atoms) may be solved without approximations, but it fails to predict electron spin and leads to models with nonsensical consequences such as negative energy states of the vacuum, infinities, and negative kinetic energy" [25]. Effort in finding solution to the Dirac and Schrödinger evades the effect of interelectron repulsion in multielectron atoms with the result that results obtained are approximations [25]. These notwithstanding a general method for solving Schrödinger and Dirac equations have been discovered [26]. The paper neither revealed application of the theories to the calculation of radii of atomized elements nor is concerned with how it can be used to calculate Bohr's radius which otherwise would have aided this research given that the nonclassical nature of Schrödinger and Dirac formalism may predict the radii of hydrogenic atoms.

I wish to add that the interpretation of Heisenberg Uncertainty Principle has also met reservation: "It is wrongly interpreted as: the uncertainty in the measured momentum times the uncertainty in the measured position must be no less than $\hbar$ as given by the equation $\Delta x \Delta \mathrm{p} \geq \hbar / 2$. The Heisenberg uncertainty principle (HUP) is the mathematical expression for the statistical error in the variables of the wavefunction such as those assigned to the position and momentum of the electron. Since the wavefunction is interpreted as the probability of the position of the electron which puts it everywhere at once with an infinite number of positions and energies simultaneously including ones with negative kinetic energy, the Heisenberg uncertainty principle merely reveals that this model (but what model?) is not a valid physical description of the electron"[25]. The common ground between the two models, classical and quantum mechanics is the understanding that an electron be it for hydrogenic and multielectron atom has both potential and kinetic energy components. There is also the issue of Bohr's introduction of the quantum concept in terms of what is referred to as an ad-hoc Planck constant [12]. It cannot be over emphasized that this research is not about wave treatment of all hydrogenic atoms and multielectron atoms.

Wave description of an electron requires that the electron has to be seen as a wave while as a particle it has to be amenable to classical Newtonian mechanics which results in Bohr's equation and the derived equation for the calculation of not just the radius of hydrogen otherwise called Bohr's radius but for multielectron atoms. The relevance that one can consider is the issue of the highest probability of locating an electron; whatever the probable position, the energy needed to remove such electron depend on its kinetic energy which is a function of the reciprocal of the distance between the electron at an uncertain position according to Heisenberg, and the nucleus.

\section{Conclusion}

An alternative equation to Bohr's radius was successfully derived. Both original Bohr's equation and derived equation can give similar result for the radius of hydrogen; the derived equation is more straightforward in its application to any atom. Therefore, Bohr's equation stands scientifically justified. Further research may be needed in order to formulate a model that can be used to determine the distance of inner electron from the nucleus of any atom in its ground state.

\section{References}

[1] Kragh H. The Early Reception of Bohr's Atomic Theory (1913-1915): A Preliminary Investigation. RePoSS: Research Publications on Science Studies 9. Aarhus: Centre for Science Studies, University of Aarhus 2010; url: http://www.css.au.dk/.

[2] Zoolman D. Bohr's model of the atom draws critics. In Kim Rendfeld. History of science. 2016.

[3] Weinberger P. Niels Bohr and the dawn of quantum theory. Philos Mag. 2014; 2-16.

[4] Barkla CG. The spectra of the fluorescent Röntgen radiations. Phil. Mag, 1911; 22, 396-412.

[5] Schwarz WHE. $100^{\text {th }}$ Anniversary of Bohr's model of the atom. Angew. Chem. Int. Ed. 2013; 52: 2-13.

[6] Sala O, Araki K. and Noda LK. A. Procedure to obtain the effective nuclear charge from the atomic spectrum of sodium. J. Chem Educ. 1999; 76 (9): 1269-1271.

[7] Pillai SO. Solid state physics. $6^{\text {th }}$ ed. New Delhi. Bangalore. Chennai. Cochin. Guwahati. Hyderabad. Jalandhar. Kolkata. Lucknow. Mumba I. Ranchi: New Age International Limited, Publishers; 2005.

[8] Lee JD. A. new concise inorganic chemistry. $3^{\text {rd }}$ ed. New York. Cincinnati. London. Toronto. Melbourne: Van Nostrand Reinhold Company Limited; 1977.

[9] Kneen WR, Rogers MJ Wand Simpson P. Chemistry. Facts, patterns, and principles. $1^{\text {st }}$ ed. London: The English Language Book Society and Addison-Wesley Publishers Limited; 1972.

[10] David R. and Lide Ed "CRC Handbook of Chemistry and Physics, CRC Press. Boca Raton, Florida, 84 ${ }^{\text {th }}$ Edition" 2003.

[11] Busch P, Heinonen T. and Lehti P. Heisenberg's uncertainty principle. Phys Rep. 2007; 452(6): 155-176.

[12] Daywitt WC. The Compton Radius, the de Broglie radius, the Planck constant, and the Bohr orbits. Prog Phys. 2011; 2:32-33.

[13] Lang PF and Barry BC. Methods of calculating ionization energies of multielectron (five or more) isoelectronic atomic ions. Sci World J. 2013; 2013: 1-11. 
[14] Lang PF and Barry BC. Relativistic corrections for calculating ionization energies of one - to five - electron isoelectronic atomic ions. ISRN Inorg Chem. 2013; 2013:1-11.

[15] Haendle BL. Presenting the Bohr atom. J. Chem Edu. 1982; 59(5): 272-276.

[16] Hettema H. Bohr's theory of the atom, 1913-1923: A. case study in the progress of scientific research programmes. Studies in history and philosophy of science Part B: Studies in history and philosophy of modern physics. 1995; 26(3): 307323.

[17] Duchowicz PR and Castro EA. Comparison between two different approaches toward atomic radii. Kragujevac J. Sci. 2005; $27: 5$ 1-56.

[18] Owolabi TO, Akande KO and Olatunji KO. Estimation of the atomic radii of periodic elements using support vector machine. IJAIST 2014; 28(28): 39-49.

[19] Ghosh DC and Biswas R. Theoretical calculation of absolute radii of atoms and ions. Part1. The atomic radii. Int J Mol Sci. 2002; 3: 87-113.

[20] Ghosh DC and Biswas R. Theoretical calculation of absolute radii of atoms and ions. Part 2 . The atomic radii. Int J Mol Sci. 2003; 4: 379-407.
[21] Islam N. and Ghosh DU Spectroscopic evaluation of the atomic size. Open Spectrosc J.2011; 5: 13-25.

[22] Islam N. Relationship between atomic orbital exponent and atomic hardness. The Sci Tech, J Sci Tech. 2012; 1(1): 40-50.

[23] Islam N. and Jana A, A. new formula for the evaluation of the ionization energy based on the orbital exponents of the atoms of 118 elements of the periodic table. J Theor Comput Chem. 2010; 09 (637): 637-651.

[24] Anonymous. Helium atom, many-electron atoms, variational principle, approximate methods, spin 2011; Accessed 24, Jan, 2017. Available: https://www1.udel.edu.

[25] Mills RL. The fallacy of Feynman's and related arguments on the stability of the hydrogen atom according to quantum mechanics. Annales de la Fondation Louis de Broglie. 2005; 30(2): 129-149.

[26] Nakatsuji H. Discovery of a General Method of Solving the Schrodinger and Dirac Equations that opens away to accurately predictive quantum chemistry. Acc. Chem. Res. 2012; 45(9): 1480-1490. 